\title{
Hardware/Software Verification Process through Cloud Computing
}

\author{
Ousama Esbel and Ng Ah Ngan Mike Christian
}

\begin{abstract}
For the past decade, we have seen an introduction of hardware/software automated systems in our community, which offer new and interesting advantages to the associated sides. However, it has become less adopted because it exposes the organizations or individuals to a variety of difficulties and limitations. Moreover, the traditional communication methods such as Bluetooth, Wi-Fi and GSM network, which are used to consolidate hardware with software limits the system functionality and scalability.

This paper proposes a new approach for integrating hardware with software through cloud computing. Utilizing cloud computing as the network backbone produces better opportunities and best practices to organizations and individuals over traditional communication methods. The paper will demonstrate how a microcontroller can be connected to a handheld device through cloud as well as providing solutions for mapping related devices and securing their channels from other devices in the system.
\end{abstract}

Index Terms-Android, Arduino, cloud computing, hardware/software integration, verification process.

\section{INTRODUCTION}

With the meteoric rise of mobile device proliferation and the human nature that prefers to have things around them being managed and controlled smoothly, many organizations and individuals have developed automated systems based on hardware/software integrations, mainly using microcontrollers. These kinds of system have changed the world around us in many aspects whether as a business value or for charity purposes [1].

Automated Systems can centralize the operations and decrease the effect of human factor, as reliance on human working force requires longer time to discover faults, deploy solutions and resolve problems, whereas automated systems can function with accuracy, versatility and with almost no errors [2].

\section{A. Existing Hardware/Software Automated Systems}

Due to the various communication interfaces that can be utilized as the network backbone to consolidate hardware with software such as, Wi-Fi, NFC, Bluetooth and RFID, many automated systems with same purpose can be generated uniquely with its own pros and cons. For instance, Home automation system (HAS), which is also known as Smart Home is one of the most anticipated systems that has been integrated through many types of communication methods.

Manuscript received September 9, 2014; revised January 7, 2015.

The authors are with Limkokwing University for Creative Studies with the department of Information Technology, Mauritius (e-mail: uzi0espil@gmail.com,mike@limkokwing.com.mu).
HAS brings some level of actions to the home environment and "provide convenience, comfort, security and energy efficiency to its occupants" [3].

Adopting Android and Arduino as HAS system's peripherals has helped to overcome the dilemmas that exist in traditional HAS as illustrated in [4]. The study in [5] has utilized Bluetooth as the network backbone, the project was meant to deliver a cheap HAS system that is affordable by the majority of people. The Bluetooth module used is a Class 1. It has frequency of $2400 \mathrm{~Hz}$ and it is able to provide connectivity up to 100 meters at speed of $3 \mathrm{Mbps}$. However, some Android devices which power Class 2 Bluetooth module has a range of 10 meters only, as known; Class 2 will limit the transmission distance of class 1 to approximately 32 feet regardless of the objects between the associated devices that can weaken the signal [6]. This shows that Bluetooth is inconvenient for most of automated systems due to the limited range of operation.

Using Wi-Fi to consolidate hardware with software can overcome the issues that have been illustrated in Bluetooth based systems. According to [5], using Wi-Fi technology can increase the ability of upgrading and reconfiguring the system, however, it will be slightly more expensive than Bluetooth-based systems. The system proposed by [5] utilizes Arduino WiFly shield module, which has the ability to connect to $802.11 \mathrm{~b} / \mathrm{g}$ wireless network. $802.11 \mathrm{~b}$ protocol uses $2.4 \mathrm{GHz}$ ISM band that can cover range of 150 feet unidirectional with speed of $11 \mathrm{Mbps}$ [6]. A Wi-Fi booster can be added to increase the coverage or by adopting XBee Wi-Fi shield that uses $802.11 \mathrm{~b} / \mathrm{g} / \mathrm{n}$ protocols which will cover up to 175 meters. However, controlling the appliances is still limited to the range of operation.

In order to break the distance limitation of locally controlled systems, the proposed system in [7] has introduced a system based on GSM network technology for transmission of SMS, where GSM shield can be plugged and developed easily with Arduino microcontroller. In spite of successfully breaking the distance limitation and reducing complexity and expense of the system, having the user to pay long-term cost of credits isn't fully accepted. In addition, the user still needs to memorize many commands to perform operations because it doesn't provide a friendly GUI. Moreover, it generates huge security risks, if an outsider got the microcontroller's SIM number and knows the controlling commands then the outsider will be able to control and break into the house.

With the emergence of Internet of Things (IoTs), researchers in [8]-[10] have presented the use of web services in HAS systems, SOAP and RESTful are used as interoperable application layer to remotely access HAS and establish a communication between the devices. The system proposed in [10] utilizes RESTful based web services as an interoperable application layer for communication between 
the remote user and the home appliances, using Ethernet or Wi-Fi shield in Arduino allows it to read or post REST data of JSON. These shields supports TCP/IP stack and the system establishes a connection with Local Area Network (LAN) using static IP address rather than acquiring an IP via DHCP. However, the consumer should configure the router to port forward the related packets to Arduino, and since the public IP address isn't fixed and a new IP address will be assigned whenever a connection to the Internet is established, then the consumer must always provide the IP address in the Android application. This dilemma is a huge drawback to the system because simply the user must get the IP address every time it changes which will affect the real-time notification due to the dynamic change of IP address.

The above-mentioned systems can be divided into two categories, Locally Controlled Systems (LCS) and Remotely Controlled Systems (RCS). LCSs are governed by the range of mobility, where the user can only control the hardware within the limited range of operation but these systems have a simpler applied server to process and control. On the other hand, RSCs allow the user to control hardware anywhere as long as a connection is established between them via Internet or GSM but the applied server is complicated and increase the expense of the system.

Regardless of the range of mobility and complexity, both categories are difficult to maintain and fix. What if the system need to be maintained due to a bug or upgraded to add a new feature, then you will need to go to each and every location you have installed the system to fix or upgrade the system's server. This issue indicates that these methods lack scalability and flexibility and increase the expense of the system.

This problem can be tackled by providing a single server to handle the storage, processes and delivery of data to all implemented systems and move it to the Internet to allow a remote control to the peripherals. In short, consolidating hardware/software through Cloud Network. By doing Thus, the system's expense will drastically decrease because installing a server to each consumer is no longer needed and less working force will be needed to maintain the system.

\section{Cloud Computing}

\section{A. Overview}

In order to shed the light on the pros and cons that Cloud Computing provides to the automated system, we need to understand what Cloud is. Cloud is a metaphor that refers to the shifting of web-based and non-web-based application to a shared data center [11]. Moreover, Cloud computing follows multi-tenancy architecture [12], it allows multiple instances to use the same infrastructure at the same time where they can customize it to their specific needs without affecting other users "tenants".

With Cloud Computing, you eliminate the need of complicated software stack and its licenses, hardware, network resources and a team of experts to install, configure, test, run, secure and update the system. Cloud provides a huge workload shift, which reduces the pressure and resources on the local computers.

\section{B. Related Work}

Researchers have already proposed Cloud network to consolidate hardware and software in automated systems. Reference [2] has proposed a HAS system that employs the integration of android device, cloud networking, wireless communication and power-line communication to provide the ability to control the house appliances. The system consists of 3 main parts:

1) Cloud Server - centralize storage, processes to give complete control over the versions of the browser-based applications provided by clients, which removes the need for installing and purchasing software's licenses on individual client computing devices.

2) Client - user's remote device that will be used to control the hardware in the house.

3) Hardware - is an 89C51 I Microcontroller with ADCs (Analog-to-Digital converters) provides similar feature to Arduino.

The system has made worthy contribution to the design and development of HAS systems. However, the existing work were mainly concentrated on switching and managing home appliances rather than explaining the functionality used to map the related devices together and differentiate the channel of sub-system devices to the others.

\section{PROBLEM STATEMENT}

Although Cloud Computing provides best practices over traditional automated systems, it still not straightforward to develop where verification process and NAT traversal are both considered a huge bottleneck to the system. The world is still depending on IPv4 that uses 32-bit address scheme allowing for a total of $2^{32}$ (just over 4 Billion addresses). With the growth of Internet and the emergence of IoTs, the number of unused IPv4 addresses is expected to drain [13].

In this case, each IP node on a network that has globally unique IP address can have many private (internal) IP addresses to provide additional range of addresses to organization and to prevent the depletion of public addresses. Private addresses are not routable on the public Internet, but are shared and accessed through the internal network only. However these devices can access the Internet by sharing one public address through the application layer gateway.

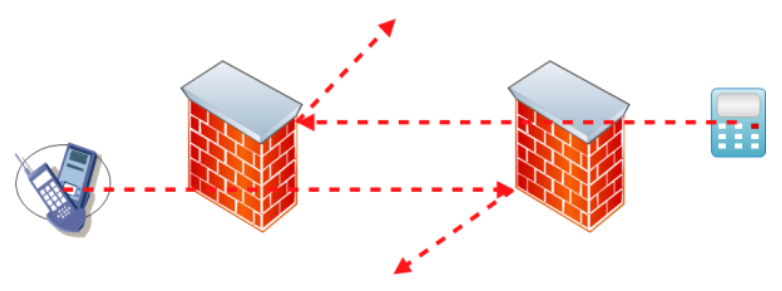

Fig. 1. Firewall demonstration.

These arrangements have made configuring a connection between devices that resides in different network difficult. The problem is most carriers in public Wi-Fi has Firewall placed. Firewalls are designed to prevent unwanted communication from reaching devices connected to the network. Presumably, Firewall cannot be accessed which means that the ports cannot be manually opened to allow incoming connections. Therefore, any incoming communication will be blocked by the Firewall and will not 
be able to communicate with the related device as illustrated in the Fig. 1 below.

In our case, Arduino microcontroller will reside in a network with a private IP address that is not directly accessible by mobile phone.

Another point that must be considered is that public IP addresses change dynamically. Each time a new connection to the Internet is established, a new IP address will be assigned from the ISP. Demanding a static IP address from the consumer or asking the consumer to configure the router to do port forwarding to the hardware is unprofessional call to do business regardless of the risks that can be generated.

\section{SOLUTIONS}

There are quite some methods to address the IP issue as shown in [14]. However, this paper will concentrate in addressing the solutions that enable connection between Android and Arduino devices specifically. Each of the following solutions has its advantages and disadvantages.

Essentially, we are trying to find a way to verify the Android device with its related Arduino and differentiate them from other sub-systems by mapping the related devices together to have an open channel for bidirectional communication as long as the Internet connection is established.

\section{A. Relay Server}

Since Firewall allows outgoing communication but doesn't allow incoming connections, then by introducing a middleman that has no Firewall or Firewall that can be accessed and controlled, we can establish a connection and open a channel between related devices. This middleman is the Relay Server.

Relay Server enables secure, load-balanced communication between associated devices, it simply receives unicast streams from the Internet and multicast them to a Local Area Network (LAN).

Relay Server accepts incoming connection from multiple devices, which means that devices can talk to the Relay Server and the Server can talk back to them. When this bidirectional communication between the server and the device has been established, the Relay Server can run an algorithm to identify and relate the device to the user's other device, and the server can bridge the connection between the related devices by sharing their ports among them which is known as port mapping.

Inspired from [15], a deeper look on how Relay Server can establish a communication between hardware and software is defined. The network consists of a set of nodes interconnected by virtual links in an arbitrary topology, which form the Cloud Each node execute a process that is called routing daemon each maintains a list of devices available to the system. Fig. 2 shows a sample network consists of 5 nodes. The continuous line represents the virtual links between the nodes while the dotted lines connect the nodes to their devices set.

The flow of the model can be demonstrated as the following: if the user wants to communicate to his Arduino though his phone, the Relay Server on the left must first find the route or path to its related device on the right. Then, it must forward user's command to each node along the path to the other Relay Server that has the user's Arduino connected to, which can then send the command to Arduino.
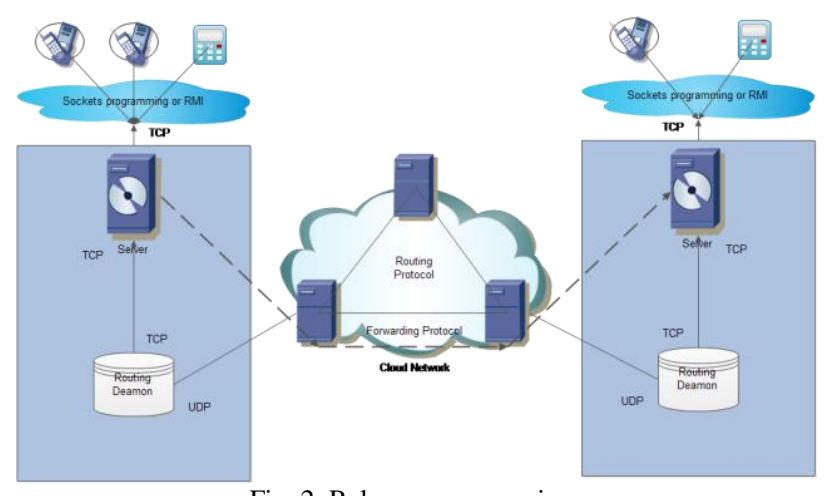

Fig. 2. Relay server overview.

The devices establish connection to the server by using Socket programming specifically Web Sockets. Socket Programming is a computer network programming, which involves writing computer network. As long as the connection is established, the channel between the server and the device is open allowing a bidirectional exchange of data and no need to worry about Firewall or port forwarding.

Fortunately, Arduino is capable of being a Client or a Server by using a Socket Programming provided by Ethernet Shield. Therefore, this method can be applied to both Android and Arduino.

In conclusion, Relay Server is a cost effective way to integrate different devices through a common, secure gateway for data transmission.

\section{B. Hosted Services}

The urge to have production systems that adapt with agile development and allow easy reconfiguration has led to the ever growing interest in automated paradigms. It provides model and services to an implemented production system that both will collaborate in a complicated manner to attain a certain goal [16].

That is why many organizations have implemented a Service-Oriented Architecture (SOA) to provide the necessary functionality and device interoperability to other organizations' automated systems. SOA is an architectural paradigm that adds functionalities to independent systems. It only exposes the required interface to the organization, and encapsulates and hides its own implementations.

Hosted Services are adopting the same concept of SOA but with addition of Event-Driven architecture forming Event-Driven SOA or known as SOA 2.0. These systems provide conveniences of information being instantly pushed between associated devices, simply because we live in a world where information is pushed to us.

Various organizations provide these services; IBM Cloud, Google Cloud and HP CM all were the first who started this trend. There are quite many hosted services that support mobile platforms especially Android platform. However, Arduino lacks the communication with most of them. Some well-known Hosted Services that can ease the functionality to consolidate Android with Arduino are:

1) Facebook Parse - A Cloud Company that hosts backend services for app developers, bought by Facebook in April 
2013 to add more advantages in terms of scalability and efficiency. Parse is capable to integrate with all kinds of mobile platform, web-browsers and any device that is capable to utilize RESTful communication like Arduino.

2) Pusher - Hosted Service to provide infrastructure of service offering real-time infrastructure for communication between your application and related devices/clients where clients could be web-browsers or mobile devices or anything that can perform TCP/IP communication.

3) Google Cloud Messaging (GCM) - service that permits bidirectional transmitting data from server to users' Android-powered device and vise-versa. The GCM will handle all the processes of queue the messages and deliver them to the target device. This feature has replaced the Cloud to Device Messaging Service (C2DM) in Android.

GCM is completely free no matter how many messages transmitted to the targets allowing $4 \mathrm{~KB}$ payload per message. On the other hand, the other Hosted Services require fees to benefit from their services; the fees vary depending on the number of opened channels or the size of messages. Unfortunately, GCM cannot establish a connection to Arduino directly. However, the free of charge service is tempting and still; by including this service the system expense will be reduced.

Fig. 3 outlines the general architecture adopted by Hosted Services. Most of Hosted Services use Web Sockets in Services layer, which provides best solution to real-time bidirectional communication between associated devices. On the past, there were hacky solutions buried under the term Comet, some of these technologies are still in use. However, Web Sockets were designed so that Comet solutions were made obsolete [17].

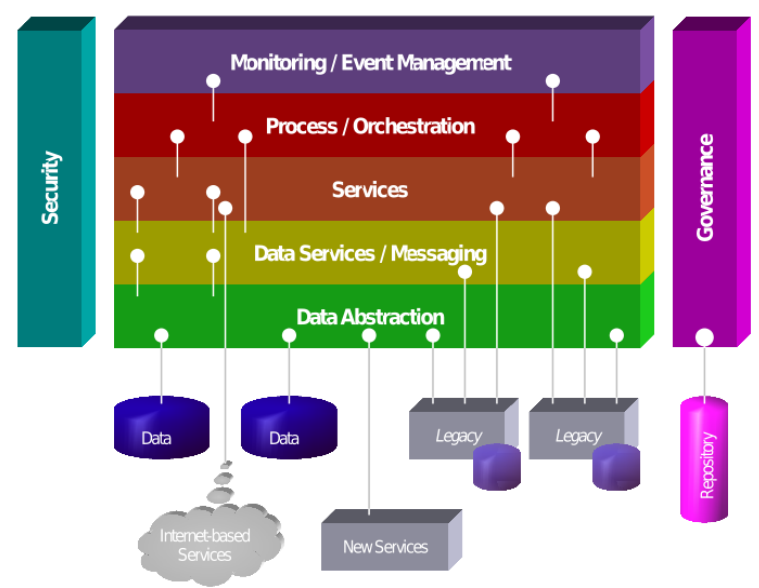

Fig. 3. Hosted services overview.

As shown in the Figure, there are a layer of Messaging and Abstraction on top of Web Sockets allowing consumer's device to use publish-subscribe paradigm. This allows the device to receive and publish from a specific channel. Therefore, connect user's Android device to the user's Arduino microcontroller through the sub-system's channel only.

The benefits from adopting Event-Driven SOA at the level of communication between devices is crucial for organizations who seek fast and effective way to consolidate hardware with software using minimal amount of resources.

\section{Software-to-Software}

Consolidating Android with Arduino was possible since the release of Android because of the various common communication interfaces that both shares like, Wi-Fi, Bluetooth, etc... Nonetheless, the consolidating was boosted drastically by the integration between Google and Arduino that has been announced in 2011 Conference [18]. During the Conference, Google has announced a new method to integrate Android with Arduino through USB Host and revealed a new development kit known as ADK. ADK is a microcontroller development board that follows the simple Open Accessory Standard Protocol created by Google. Although there were other board designs known to be ADK compatible, Google has made this move because Arduino's community has grown tremendously in the last few years.

As we saw, mapping the related devices has been the bottleneck of the system and it has always been easier to consolidate devices with same background together than consolidating different devices. By using ADK, Android device can be used to communicate directly with Arduino through USB cable.

Thus, in order for the user to control the microcontroller, the user will send commands from his Android mobile phone to the other Android device that is attached to the microcontroller by USB cable. Then whenever the attached Android gets the commands, it will push the commands to the microcontroller through USB.

In this case, the Attached device will act as the middleman between the sender and receiver, by using Hosted Services like GCM it will be easier to have an open channel between the related devices using XMPP feature that will eliminate the need to have $3^{\text {rd }}$ party server.

Fig. 4 illustrates the flow of information between the peripherals, where Arduino gives commands to the attached Android and distribute it to the other devices.

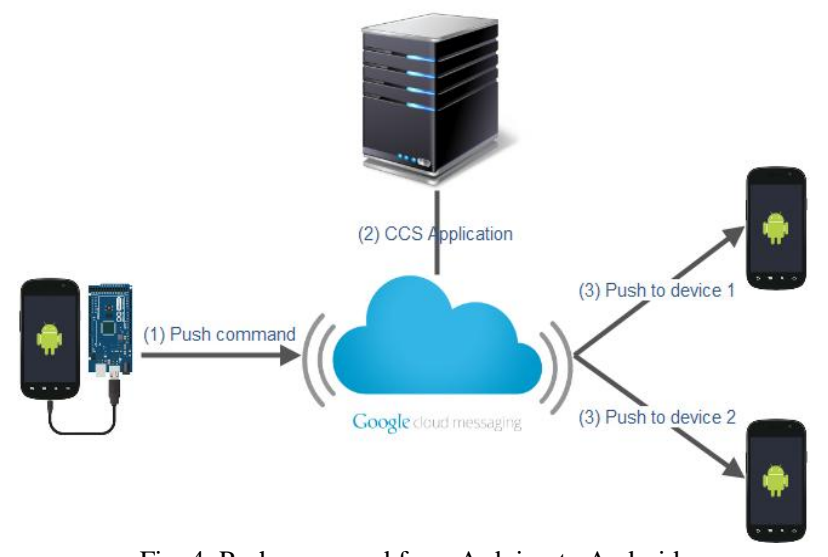

Fig. 4. Push command from Arduino to Android.

Unfortunately, this method is only applicable to microcontrollers that have compatibility with Android ADK, yet this solution provides an easy and effective way to consolidate both hardware and software as a whole unit.

\section{COMPARISON}

The architecture provided by the mentioned methods 
allows the devices in the automated system to play both roles the correspondent and the receiver [19], thus enabling peer-to-peer interactions. The general scheme that has been adopted in these methods:

1) Addressing - The foundation of the system's network that refers to the participated devices' IP addresses that provided by IP protocol, assigned by either DHCP or statically.

2) Discovery - When the device becomes part of the network, an algorithm will be followed to identify the related device and send a discovery message containing essential information to the other device check if it is online and map the devices together.

3) Description - Once the discovery phase has been successfully conducted, the related devices' information and metadata (Description) will be distributed among them.
4) Control - this phase indicates that the related devices are connected and they are aware of each other. In this phase, any of the related devices can send control commands to the other device.

5) Event Notification - layer usually implemented by publish-subscribe mechanism, allowing related devices to "subscribe" to a certain channel and publish or listen for "publishes" by other related devices. In short, devices communicating through asynchronous event channels.

The problem is with Discovery, establishing a secure, open channel for devices to communicate and eliminate the need for port forwarding or static IP address. The solutions provided in this paper are summarized in the following Table I:

TABLE I: COMPARISON BETWEEN THE PROVIDED SOLUTIONS

\begin{tabular}{|c|c|c|c|}
\hline \multirow{2}{*}{ Criteria } & \multicolumn{3}{|l|}{ Solutions } \\
\hline & Relay & Hosted Services & Software-to-Software \\
\hline Complexity & $\begin{array}{l}\text { The most complicated. Building the system } \\
\text { from the scratch or setting up a one isn't an easy } \\
\text { job. }\end{array}$ & $\begin{array}{l}\text { Less complicated, depend on what provider } \\
\text { has been chosen for the integration, because } \\
\text { each provider has their own way to integrate } \\
\text { which vary in complexity term. Yet it is } \\
\text { much easier than setting up a Relay Server. }\end{array}$ & $\begin{array}{l}\text { Least Complicated. Using GCM } \\
\text { XMPP feature can even eliminate } \\
\text { the need of server. }\end{array}$ \\
\hline Scalability & $\begin{array}{l}\text { Huge scalability. Having system that has been } \\
\text { developed in-house or hybrid of Insourcing and } \\
\text { outsourcing allow you to be totally in control of } \\
\text { the system. Thus, it can be scaled up or down as } \\
\text { needed. }\end{array}$ & $\begin{array}{l}\text { Less scalability. Outsourcing the system } \\
\text { provides lack of control in the status of } \\
\text { development and dependency on a specific } \\
\text { supplier where you can only control the } \\
\text { service interface provided to you. }\end{array}$ & $\begin{array}{l}\text { Least scalability. GCM doesn't } \\
\text { provide any interface for the } \\
\text { organizations' personnel to access } \\
\text { and control the service. }\end{array}$ \\
\hline Security & $\begin{array}{l}\text { Very secure. Since the system has been } \\
\text { developed in-house then it depends on the trust } \\
\text { of the employees and how automated are the } \\
\text { services. }\end{array}$ & $\begin{array}{l}\text { Less secure. The server used to process the } \\
\text { data resides on the chosen outsourcing } \\
\text { company. Thus, it depends on how far can } \\
\text { you trust them and how automated are their } \\
\text { services. }\end{array}$ & $\begin{array}{l}\text { It has been pointed that GCM } \\
\text { should not be used to send } \\
\text { sensitive data. So basically, it } \\
\text { depends on whether you trust } \\
\text { Google or not. }\end{array}$ \\
\hline Other Points & $\begin{array}{l}\text { Needs far more employees to develop, deploy, } \\
\text { test and maintain the system. }\end{array}$ & $\begin{array}{l}\text { Need to find the best Hosted Service that } \\
\text { can integrate the suitable hardware and } \\
\text { software because not all Hosted Services } \\
\text { can provide them. Also, understanding the } \\
\text { privacy and security provided by them is } \\
\text { important. }\end{array}$ & $\begin{array}{l}\text { Works only on devices that are } \\
\text { compatible with GCM and ADK. } \\
\text { Also, a strategy is needed to } \\
\text { maintain the power and battery } \\
\text { level of the attached device. }\end{array}$ \\
\hline
\end{tabular}

\section{CONCLUSION}

Using Cloud Computing as network backbone in hardware/software automated system will boost the system and add variety of advantages such as, increase scalability, flexibility and decrease the expense of the system. Also by centralizing the storage and providing single server to handle the mapping and connections of the implemented sub-systems, it will be easier to maintain the system, enhance the security and reduce duplications and expenses.

Although Cloud Computing provides many enhancements over traditional automated systems, it is still not easy to develop, where verification process and NAT traversal are both considered a huge bottleneck to the system. Allowing real-time communication with related devices, an established open channel is needed however, Firewall allows only outgoing communications and prevents any incoming connections, then establish such channels are not seem possible.

By introducing a middleman between the server and the client that allows a bidirectional communication between the associated devices the Firewall persistence can be solved.
Basically, the middleman accepts multiple connections from the devices and follows a specific algorithm to identify and relate the device to the user's other devices. Afterwards, the server will bridge the connections and do port mapping.

The middleman could be Relay Server or Hosted Services that has no Firewall or a Firewall that can be configured and controlled. Choosing either of them depends on the system's vision, scalability and the money invested to build the system.

Relay server is a bit more complicated however, it provides better accessibility and scalability to the system, and it also provides better security measurements. On the other hand, the API provided by the chosen Hosted Services makes the server less complicated and easier to develop but it affects the scalability and accessibility to the data.

As for the future work, Integrating Android with Arduino via Cloud Network will be conducted by developing a Relay Server to map and verify the sub-system's devices and isolate its channel from other sub-systems.

\section{REFERENCES}

[1] Wikipedia. (May 8, 2014). Automation. [Online]. Available: http://en.wikipedia.org/wiki/Automation\#Advantages_and_disadvant ages 
[2] M. B. Salunke, D. Sonar, N. Dengle, S. Kangude, and D. Gawade, "Home automation using cloud computing and mobile devices," IOSR Journal of Engineering, vol. 3, pp. 35-37, 2013.

[3] Wikipedia. (May 10, 2014). Home automation. [Online]. Available: http://en.wikipedia.org/wiki/Home_automation

[4] A. ElShafee and K. A. Hamed, "Design and Implementation of a WiFi based home automation system," World Academy of Science, Engineering and Technology, pp. 2177-2180, 2012.

[5] R. A. Ramlee, M. H. Leong, R. S. S. Singh, M. M. Ismail, M. A. Othman, and H. A. Sulaiman, "Bluetooth remote home automation system using Android application," The International Journal of Engineering and Science, vol. 2, pp. 149-153, 2013.

[6] Wood. Difference between a class $1 \&$ Class 2 bluetooth adapter. Everyday Life. Demand Media. [Online]. Available: http://everydaylife.globalpost.com/difference-between-class-1-class-2 -bluetooth-adapter-33512.html

[7] C. Felix and I. J. Raglend, "Home automation using GSM," in Proc. the 2011 International Conference on Signal Processing, Communication, Computing and Networking, IEEE, 2011, pp. 15-19.

[8] A. Kamilaris, V. Trifa, and A. Pitsillides, "HomeWeb: An application framework for web-based smart homes," in Proc. 2011 18th International Conference on Telecommunications, IEEE, 2011, pp. 134-139.

[9] S. Mukherjee, S. N. Boris, Y. Thushyanthan, and K. Gajan, "Android based farm automation using GR sakura," Sona College of Technology, 2013.

[10] R. Piyare, "Internet of things: Ubiquitous home control and monitoring system using Android based smart phone," International Journal of Internet of Things, pp. 5-11, 2013.

[11] Wikipedia. (July 12, 2014). Cloud Computing. [Online]. Available: http://en.wikipedia.org/wiki/Coud_computing

[12] M. Pathirage, S. Perera, I. Kumara, and S. Weerawarana, "A multi-tenant architecture for business process executions," in Proc. 2011 IEEE International Conference on Web Services, 2011, p. 121, 128.

[13] Webopedia. (July 23, 2014). What Is The Difference Between IPv6 and IPv4? [Online]. Available: http://www.webopedia.com/DidYouKnow/Internet/ipv6_ipv4_differe nce.html
[14] R. D. Caytiles and B. Park, "Mobile IP-based architecture for smart homes," International Journal of Smart Home, vol. 6, pp. 29-36, 2012

[15] 15-441: Computer Networks Project 1: Internet Relay Chat (IRC) Server Lead TA: Wittawat Tantisiriroj Assigned, August 31, 2010

[16] A. Fox, R. Griffith, A. Joseph, R. Katz, A. Konwinski, G. Lee, D. Patterson, A. Rabkin, and I. Stoica, "Above the clouds: A berkeley view of cloud computing," Dept. Electrical Eng. And Comput. Sciences. University of California, Berkeley, Rep. UCB/EECS 28 , 2013

[17] Wikipedia. (July 11, 2014). WebSocket. [Online]. Available: http://en.wikipedia.org/wiki/WebSocket

[18] Slovati. September 2001 on Google chooses Arduino as a development platform for Android. [Online]. Availuable: http://dev.emcelettronica.com/google-chooses-arduino-developmentplatform-android

[19] F. Jammes, A. Mensch, and S. Harm, "Service-oriented device communications using the devices profile for web services," in Proc. the $3^{\text {rd }}$ international workshop on Middleware for pervasive and ah-hoc computing, ACM, 2005, pp. 1-8.

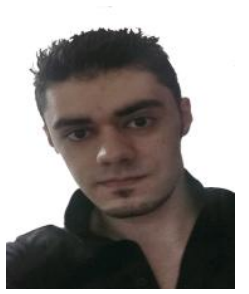

Ousama Esbel was born on March 4, 1992 and raised in U.A.E, but he is originally from Syria. He is pursuing his B.E degree in information technology in Limkokwing University of Creative Technology, Malaysia, Selangor. His areas of interests are distributed systems, robotics and networking.

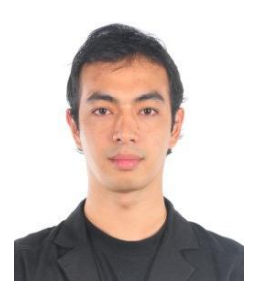

Ng Ah Ngan Mike Christian was born on July 12 , 1983, and raised in Mauritius. He got his BSc in software engineering with Multimedia, Limkokwing University of Creative Technology, Malaysia, Selangor. His areas of interests are mobile development and augmented reality. 